\title{
Suturing extraction sockets on patients maintained on oral anticoagulants
}

\author{
Is suturing necessary after dental extractions when patients temporarily \\ discontinue their anticoagulant therapy?
}

\author{
Al-Mubarak S, Al-Ali N, Rass MA, et al. \\ Evaluation of dental extractions, suturing and INR on \\ postoperative bleeding of patients maintained on oral \\ anticoagulant therapy. Br Dent J 2007; vol:203. Epub 2007 Aug 10.
}

Design A randomised controlled trial (RCT) was carried out. Intervention Patients scheduled for dental extraction were randomly divided into four groups: no suturing with either discontinued anticoagulants (group 1) or continued warfarin (group 2); and suturing with either discontinued anticoagulants (group 3) or continued warfarin (group 4). The international normalised ratio (INR) was determined at different timepoints (baseline, days 1, 3 and 7).

Outcome measure The status of bleeding and healing were assessed by an independent examiner blinded to the treatment protocol for the four treatment groups.

Results Discontinuing warfarin reduced INR level significantly at day 1 , and it subsequently reached $<1.5$ in 96 out of 104 patients (groups 1 and 3). Statistical comparisons between the different treatment groups did not reveal any significant difference regarding bleeding status or healing pattern. Interestingly, patients who received sutures showed a higher but insignificant incidence of bleeding postoperatively compared with their respective controls.

Conclusions Dental extractions may be safely performed for people who are taking anticoagulation therapy provided the INR level is kept $\geq 3.0$ and effective measures of local haemostasis are administered. The decision to suture should be made on a case-by-case basis, as the trauma associated with soft tissue handling might outweigh its advantages in some situations, such as simple extractions.

\section{Commentary}

Warfarin is a widely prescribed and highly valuable oral anticoagulant in the prevention of thromboembolic events. Dental management of patients taking this drug should take into account the potential for haemorrhage associated with anticoagulant therapy. This RCT aims to compare management regimes of patients taking warfarin who are scheduled for dental extractions.

The design of the study is generally sound, although one problem that is difficult to avoid is the partly subjective nature of assessing bleeding and healing. The authors have attempted to minimise potential bias by blinding the examiner to warfarin status and setting clear definitions for bleeding and healing, but there is no mention of a calibration process or checks of intra-examiner variability.

Address for correspondence: Dr Sultan Al-Mubarak, Consultant Periodontist PO Box 64399, Riyadh 11536, Saudi Arabia.

E-mail: smubarak@humanitariancity.org.sa
In addition, blinding of the examiner is obviously not possible to the use of sutures.

In this trial there were fortunately no incidences of thromboembolism, despite significantly reduced INR levels in the groups that discontinued warfarin. In the discussion, the authors highlight evidence demonstrating the risks of withdrawal of anticoagulant therapy. In view of the potentially serious risks involved with this, it might have been preferable if the paper had clarified whether ethical approval was granted or examiners liased with patients' medical practitioners prior to alteration of warfarin regimes.

The paper generally outlines the trial method and results clearly, but further information would have allowed a more comprehensive appraisal of the study. This should describe the patients' demographic, operative or medical variables and would be of use in identifying or excluding any possible confounding factors. Although randomisation should account for this, the method of randomisation is not discussed and there is no record of numbers lost to followup or dropout subsequent to group allocation, which could affect the supposedly even distribution of variables throughout the groups. It seems unlikely that a prospective study of 214 patients had no loss to followup. An intention-to-treat analysis could help account for any subjects lost during the trial as the analysis carried out in the paper, on complete cases only, may wrongly estimate the effectiveness of the interventions. Unfortunately, there is no discussion of intentionto-treat analysis or data to tell if this was necessary.

One method which could have made this clearer would be the inclusion of a CONSORT flow diagram which is designed to provide an overview of the flow of participants through a trial. ${ }^{1}$ In addition, the use and reporting of a power calculation would have shown if the sample size was appropriate for this study.

Because of the points discussed above, care should be exercised when drawing conclusions from the results. The trial does provide limited evidence, however, that continuing warfarin therapy in patients undergoing dental extractions has little adverse effect on postoperative healing or bleeding. Importantly, this will also avoid the increased risk of thromboembolic events associated with stopping warfarin, one of the main points emphasised in current guidelines. ${ }^{2,3}$

\section{Cameron Walker}

Glasgow Dental Hospital and School, Glasgow, Scotland, UK

1. Altman DG, Schulz KF, Moher D, et al. The revised CONSORT statement for reporting randomized trials: explanation and elaboration. Ann Intern Med 2001; 134:663-694.

2. Surgical management of the primary care dental patient on warfarin. Liverpool: North West Medicines Information Centre; 2004, revised 2007.

3. Perry DJ, Noakes TJ, Helliwell PS. Guidelines for the management of patients on oral anticoagulants requiring dental surgery. Br Dent J 2007; 203:389-393.

Evidence-Based Dentistry (2008) 9, 7. doi:10.1038/sj.ebd.6400559 